\title{
Melanoma Differentiation Associated Gene-7 (mda-7): A Novel Anti-Tumor Gene for Cancer Gene Therapy
}

\author{
Abner M. Mhashilkar, ${ }^{1}$ Robert D. Schrock, ${ }^{1}$ Mustafa Hindi, ${ }^{1}$ Jennifer Liao, ${ }^{1}$ Kerry Sieger, ${ }^{1}$ \\ Famourou Kourouma, ${ }^{1}$ X. Helena Zou-Yang, ${ }^{1}$ Eric Onishi, ${ }^{1}$ Oksana Takh, ${ }^{1}$ Thomas S. Vedvick, ${ }^{2}$ \\ Gary Fanger, ${ }^{2}$ Lorna Stewart, ${ }^{3}$ Graham J. Watson, ${ }^{3}$ David Snary, ${ }^{3}$ Paul B. Fisher, ${ }^{4}$ Tomoyuki Saeki, ${ }^{5}$ \\ Jack A. Roth, ${ }^{5}$ Rajagopal Ramesh, ${ }^{5}$ and Sunil Chad $a^{1^{*}}$ \\ ${ }^{1}$ Introgen Therapeutics Inc., 2250 Holcombe Blvd, Houston, TX 77030, USA \\ ${ }^{2}$ Corixa Inc. Seattle, WA, USA \\ ${ }^{3}$ Imperial Cancer Research Fund, Applied Development Laboratory, London, UK \\ ${ }^{4}$ Departments of Neurosurgery, Pathology and Urology, Columbia University, College of Physicians and \\ Surgeons, New York, NY, USA \\ ${ }^{5}$ Department of Thoracic and Cardiovascular Surgery, M.D. Anderson Cancer Center, Houston, TX 77030, USA \\ Contributed by A. B. Pardee. Accepted February 15, 2001.
}

\begin{abstract}
Background: The mda-7 gene (melanoma differentiation associated gene-7) is a novel tumor suppressor gene. The anti-proliferative activity of MDA-7 has been previously reported. In this report, we analyze the antitumor efficacy of Ad-mda7 in a broad spectrum of cancer lines.

Materials and Methods: Ad-mda7-transduced cancer or normal cell lines were assayed for cell proliferation (tritiated thymidine incorporation assay, Alamar blue assay, and trypan-blue exclusion assay), apoptosis (TUNEL, and Annexin V staining visualized by fluorescent microscopy or FACs analysis), and cell cycle regulation (Propidium Iodide staining and FACs analysis).
\end{abstract}

Results: Ad-mda7 treatment of tumor cells resulted in growth inhibition and apoptosis in a temporal and dosedependent manner. The anti-tumor effects were independent of the genomic status of p53, RB, pl6, ras, bax, and caspase 3 in these cells. In addition, normal cell lines did not show inhibition of proliferation or apoptotic response to Ad-mda7. Moreover, Ad-mda7-transduced cancer cells secreted a soluble form of MDA-7 protein. Thus, Ad-mda7 may represent a novel gene-therapeutic agent for the treatment of a variety of cancers.

Conclusions: The potent and selective killing activity of Ad-mda7 in cancer cells but not in normal cells makes this vector a potential candidate for cancer gene therapy.

\section{Introduction}

Adenoviral vectors harboring therapeutic genes have been used successfully for gene transfer in vitro and in vivo (1-3). The novel mda-7 gene (melanoma differentiation associated gene-7) was identified by subtraction hybridization using a human melanoma cell line (HO-1), induced to lose growth potential irreversibly and terminally differentiate after treatment with the combination of IFN $-\beta$ and mezerein $(4,5)$. Further studies showed that mda-7 mRNA was expressed in normal melanocytes and in early stages of melanoma, but was decreased or lost during melanoma progression (5). The mda-7 cDNA encodes a novel and evolutionarily conserved protein of 206 amino acids with a predicted size of $23.8 \mathrm{kD}$ (5). The protein sequence shows no significant homology to known proteins, with the exception of a small stretch that displays limited homology to

Address correspondence and reprint requests to: Dr. Sunil Chada, Introgen Therapeutics Inc., 2250 Holcombe Blvd., Houston, TX 77030, USA. Phone: (713) 610-4007.

Fax: (713) 797-1349. E-mail: s.chada@introgen.com
IL-10 (5). In earlier studies, the transfection of a mda-7 expression vector into a variety of tumor cell lines containing both wild-type and mutant p53 and $\mathrm{RB}$ genes resulted in the suppression of monolayer growth and anchorage independence (6). The lack of sequence similarity between mda- 7 and previously described growth-suppressing genes suggested that mda-7 may represent a new class of growth suppressors (5-10). Infection with Admda7 in wild-type, null, and mutant p53 human breast cancer cells induced apoptosis, which correlated with an increase in the expression of the proapoptotic protein BAX and a concomitant increase in the ratio of BAX to BCL-2 (7-10). Furthermore, ex vivo infection of the MCF-7 human breast carcinoma cells with Ad-mda7 inhibited tumor development in nude mice (7). Infection of established human cervical cancer (HeLa) xenografts in nude mice with Ad-mda7-inhibited tumor growth and progression (8-10). The inhibitory activity of Ad-mda7 in the p53 wild-type MCF-7 and p53- and Rb-inactivated HeLa cell lines is important, as these cell lines are not efficiently killed by other tumor suppressor 
genes $(11,12)$. In contrast, elevated expression of MDA-7 in normal mammary epithelial cells did not alter their cell cycle kinetics, growth rate, or survival (7). In the current study, we have evaluated the antitumor potency and selectivity of Ad-mda7, using a spectrum of tumor cell lines derived from breast, colon, and lung. We report here that Ad-mda7 suppresses cancer cell growth in a dose-dependent manner, independent of p53, RB, ras, and p16 mutational status. Moreover, in a number of human cancer cell lines, Ad-mda7 induces apoptosis without affecting BAX protein levels. Ad-mda7 transduced cells secrete a soluble form of MDA-7 protein, which may trigger a "bystander killing" effect on neighboring nontransduced cancer cells. Further functional studies are underway to characterize this soluble form of mda-7. In summary, mda-7 represents a novel cancer growth suppressing and apoptosisinducing gene with potential utility in gene therapy of human cancer.

\section{Materials and Methods}

Cell Lines and Cell Culture

All tumor cell lines used were obtained from American Type Culture Collection (ATCC, Rockville, $\mathrm{MD})$. The cell lines evaluated were as follows: breast (MCF-7, T47D, SKBr3, HBL-100, BT-20, MDA-MB-231, MDA-MB-468, MDA-MB-361), colorectal (DLD-1, SW-620, SW-480, HT-29, HCT-116, LS 174T), lung (H1299, H460, A549, H322, H358), and osteosarcoma (SaosLM2) cancer lines. Normal lines (Human Vascular Endothelial cells, HUVEC, normal human melanocytes, NHEM and Primary Human Mammary Epithelium cells, HMEC) were obtained from Clonetics (San Diego). MJ90 primary fibroblasts were obtained from O. Smith (Baylor College of Medicine). Normal human lung fibroblasts, NHLF, were obtained from MDACC. The cells were grown in DMEM medium (GIBCO/BRL, Life Technologies, Grand Island, NY) and fetal bovine serum (at 5-10\% final concentration, according to suppliers recommendation). The cells were free of mycoplasma and were used in the log phase of growth. Cells were harvested with $0.125 \%$ Trypsin -1.3 mM EDTA (GIBCO).

\section{Recombinant Adenovirus Production}

Replication-deficient human type 5 Adenovirus (Ad5) carrying the mda-7 gene was produced as described (7). The mda-7 gene was linked to an internal CMV-IE promoter and followed by SV40 polyadenylation [p(A)]. Ad-luc and Ad-CMVp(A) (luciferase and empty vector, respectively), were used as control viruses. Briefly, for the generation of Ad-vectors, Ad5 vectors harboring the gene cassettes were co-transfected with plasmid pJMl7 (13) in HEK 293 cells to rescue recombinant Ad-mda7, Ad-luc, and Ad-CMVp(A) viruses. Plaques were picked, virus stocks were grown, and their genomes were confirmed by PCR/Restriction analysis and DNA sequencing. Viruses were propagated in 293 cells and purified by HPLC.

\section{MDA-7 Polyclonal Antibodies and Western Blot Analysis}

The recombinant MDA-7 protein was expressed in E.coli and purified using a nickel NTA agarose column. This recombinant MDA-7 protein was used to generate rabbit polyclonal antisera that was further purified by affinity chromatography. This antibody was used in Western blot analyses at dilutions ranging from 1:5000-1:10,000 (from stock of $1 \mathrm{mg} / \mathrm{mL}$ ). Cell lysates $\left(10^{5}-10^{6}\right.$ cells were suspended in $500 \mathrm{uL}$ of Laemmli buffer with 5\% 2-mercapto-ethanol \{2ME\}) or supernatants (1:1 mixing with Laemmli buffer $+2 \mathrm{ME}$ ) were obtained after cells were treated with Ad-mda7 for desired length of time, followed by SDS polyacrylamide gel electrophoresis and western blot analysis using the Super-Signal substrate for Horse-Radish Peroxidase (Pierce Inc.). Other monoclonal antibodies used in the study specifically recognized BAX (Santa Cruz Biotechnology) and $\beta$-actin (Sigma).

\section{Transduction and Cell Proliferation Studies}

Cancer or normal cell lines used in this study were infected with Ad-mda7 (using $\operatorname{Ad-CMVp(A)}$ or Ad-luc as controls) with increasing MOIs (0, 100, $250,500,1000,2500,5000$, and 10,000 viral particles (vp)/cell). Cells were plated at 500-2000 cells/well in 96-well format for ${ }^{3} \mathrm{H}$-thymidine incorporation-assay, at $10^{5}-10^{6}$ cells/well in a 6 -well plate format for protein expression or apoptosis assays, or at $10^{4}$ cells/well (96-well format) for Alamar-blue assay. For infection, Ad-mda7 or Adluc (or Ad-CMVp(A)) were used at increasing titers. For ${ }^{3} \mathrm{H}$-thymidine/apoptosis, protein expression and Alamar assays, cells were analyzed 3 and 5 days postinfection.

\section{${ }^{3} \mathrm{H}$-thymidine Assay}

Growth inhibition of cells after treatment was primarily measured by analysis of incorporation of ${ }^{3} \mathrm{H}-$ Thymidine into replicating DNA. Briefly, a stock solution of $100 \mu \mathrm{Ci} / \mathrm{mL}$ of ${ }^{3} \mathrm{H}$-Thymidine (Amersham) was prepared by dilution into high-glucose DMEM (GIBCO). ${ }^{3} \mathrm{H}$-Thymidine was added to each well at a final concentration of $1 \mu \mathrm{Ci} / \mathrm{mL}$. The reaction was stopped $15 \mathrm{hr}$ later by removal of the supernatant from recipient cells. The cells were harvested by addition of 100x Trypsin/EDTA (GIBCO) to each well for $15 \mathrm{~min}$ at $37^{\circ} \mathrm{C}$. Cells were collected on a Filter using a Packard Filtermate cell Harvester following the manufacturers protocol and washed in deionized water and methanol. The filters were dried and analyzed using a Matrix 9600 (Packard).

\section{Trypan Blue Exclusion Assay}

Cell viability was measured using the Trypan blue exclusion assay. Briefly, cells were plated at the 
density of $10^{3}$ cells/chamber (Chamber slides, NUNC) and were transduced with different MOIs of Ad-mda7 (or Ad-luc, as control). After 3-5 days posttransduction, the cells were stained with $1 \%$ Trypan blue and observed under the light microscope. Three to four microscopic fields were scored for number of blue-stained cells (dead cells) to obtain the average \% killing effect of Ad-mda7.

\section{Alamar Blue Assay}

Growth inhibition of cells was also measured using the Alamar Blue assay. Briefly, cells were plated at $10^{4} \mathrm{cells} / \mathrm{well}$ in a 96 well plate format. Four days after infection with different MOIs of Ad-mda7 or control vectors, $20 \mu \mathrm{L}$ of Alamar blue dye was added to each well, and the plate was incubated at $37^{\circ} \mathrm{C}$ for 6-8 hours. The plates were then processed for optical density absorbance analysis using the Dynatech MRX plate reader at dual wavelengths of 575 and $600 \mathrm{~nm}$. Revelation 3.2 software program was used to analyze data.

\section{TUNEL Assay}

Cancer cells were seeded in Lab-Tek chamber slides (Nunc) at density of $10^{3}-10^{4}$ cells/chamber. Cells were transduced with desired concentration of Advectors. At different time points after infection, cells were analyzed for apoptosis induction using the Chromogenic TUNEL-POD (Boehringer Mannheim) assay. Cells were analyzed 2-5 days postinfection. The kit uses a Deoxythymidine transferase (TdT) enzyme to incorporate Fluorescein bounddeoxythymidine molecules to fragmented DNA with free hydroxyl groups. After washing with PBS, Horseradish peroxidase (POD)-tagged anti-fluorescein antibody is used as the secondary agent, and samples are exposed to DAB to identify TUNEL positive cells (dark brown staining).

\section{Annexin V Assay}

Using the ApoAlert Annexin V-FITC kit (CLONTECH), we also analyzed cancer cells for apoptosis. Briefly, Ad-vector-transduced cells $\left(10^{5}-10^{6}\right.$ cells total) were washed extensively in PBS and then incubated with Annexin V-FITC reagent for 30 minutes on ice. Cells were then washed and processed for FACS analysis and fluorescent microscopy.

\section{DNA Staining with Propidium Iodide (PI)}

Cell-cycle staging was done by the evaluation of DNA content by PI staining. For identifying cells at different stages of cell cycle, vector infected cancer cells were prepared as a single cell suspension of $1-2 \times 10^{6}$ cells $/ \mathrm{mL}$ of PBS. After the cells were fixed with cold $70 \%$ ethanol for $2 \mathrm{hr}$, the cells were centrifuged, the fixative decanted, and cells washed $2 \mathrm{x}$ with PBS and then stained with Propidium Iodide (PI) at final concentration of $50 \mu \mathrm{g} / \mathrm{mL}$ with RNAse at $20 \mu \mathrm{g} / \mathrm{mL}$ in PBS. Treated cells were then evaluated by FACS analysis.

\section{Confocal Studies}

Confocal microscopic studies were done at the Fluorescence Microscopy and Imaging Core Laboratory of University of Texas, Health Science Center (Houston, TX) and Imperial Cancer Research Fund Applied Development Laboratory (London, UK). In brief, H460, HeLa or MCF-7 cells were infected with Ad-mda7 (or Ad-luc) at MOI of 500 or $1000 \mathrm{vp} / \mathrm{cell}$. After 48 hours, the cells were washed $3 x$ with PBS ( $3 \mathrm{~mL} / \mathrm{wash}$ ) and treated with $500 \mathrm{uL}$ of 1:2000 diluted rabbit anti-MDA-7 affinity purified antibody (stock conc. $1 \mathrm{mg} / \mathrm{mL}$ ) at $4^{\circ} \mathrm{C}$ for $2 \mathrm{hr}$. After the treatment, the cells were washed again with PBS (3x) and treated with 1:1000 diluted goat anti-rabbit IgG-tagged rhodamine/FITC. The cells were also stained with Annexin V and Hoechst stain. Studies using HeLa cells were counter stained with Phalloidin Alexa 594.

\section{Genomic Analysis of mda-7 Coding Region}

The cDNA sequence of mda-7 was compared with the Genbank database, and seven exons comprising the mda-7 gene were identified. Exonspecific primers were prepared and the mda-7 coding region was sequenced from genomic DNA prepared from five tumor lines and 10 normal PBL.

\section{Results}

\section{Ad-mda7 Selectively Inhibits Growth of Cancer Cells}

Eight breast cancer cell lines (MCF-7, T47D, SKBr3, HBL-100, BT-20, MDA-MB-231, MDA-MB-468, and MDA-MB-361) were infected with Ad-mda7. Seven of these were strongly growth inhibited by Admda7. The two cell lines that demonstrated the highest sensitivity to Ad-mda7 were T47D (p53 mutated) and MCF-7 (p53 wild-type) (Figure 1A), as determined by ${ }^{3} \mathrm{H}$-thymidine incorporation assays. The Ad-mda7 concentration for $50 \%$ growth inhibition $\left(\mathrm{IC}_{50}\right)$ of the T47D and MCF-7 cells averaged 500 and $1500 \mathrm{vp} / \mathrm{cell}$, respectively. Furthermore, six colorectal cancer lines (DLD-1, SW-620, SW-480, HT29, HCT-116, and LS174T) were infected with Admda7. All these lines were also growth inhibited by Ad-mda7 transduction, with SW-620 and DLD-1 being the most sensitive (Figure 1B). In addition, five lung cancer lines (H1299, H460, A549, H322, and H358) were also infected with Ad-mda7. All these lines demonstrated substantial growth-inhibition by Ad-mda7. The H1299 (p53 null) and H322 (p53 mutated) cell lines were the most sensitive to Admda7 killing (Figure 1C). The $\mathrm{IC}_{50}$ in these lines ranged from $250 \mathrm{vp} /$ cell to $2000 \mathrm{vp} / \mathrm{cell}$. Of the 20 tumor lines evaluated, 19 showed substantial growth inhibition by Ad-mda7, in both temporal and dose-dependent fashions. These results significantly expand the previous study (7), in which five breast cancer and two normal breast lines were examined using a single dose of Ad-mda7. The activity of Ad-mda7 is not limited to breast cancer but 
(A) BREAST

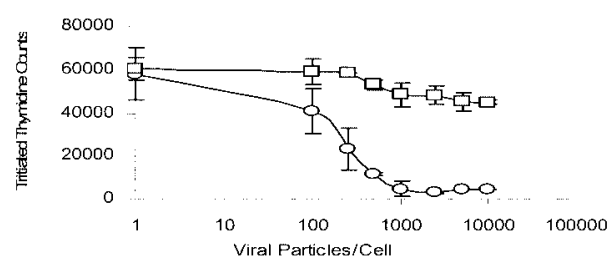

B) COLORECTAL SW620

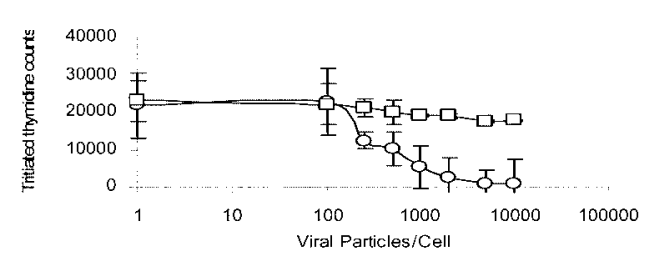

C) LUNG

H1299

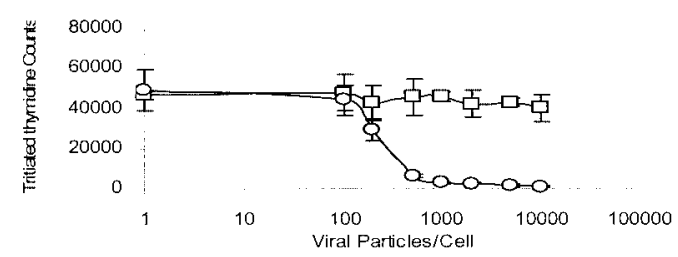

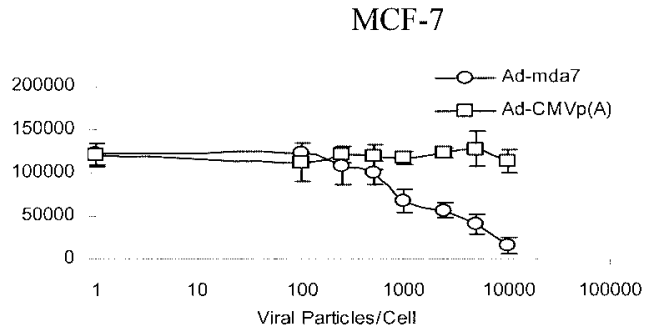

DLD-1
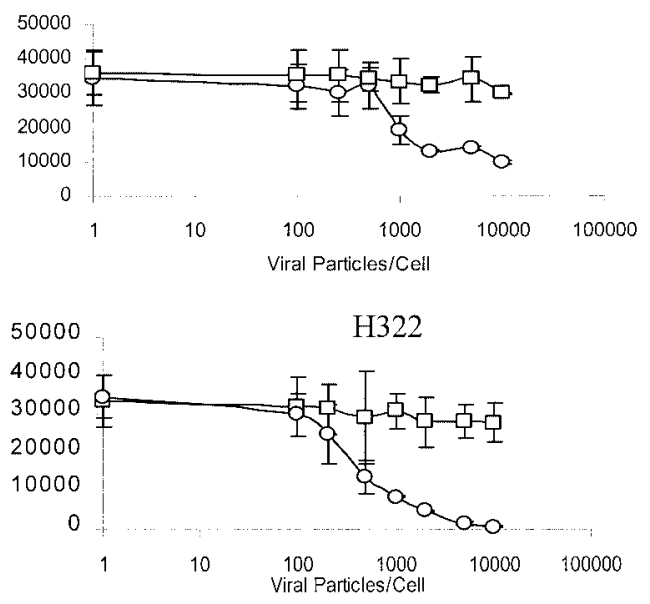

Fig. 1. Effect of Ad-mda7 on cancer cells (tritiated thymidine assay). Panel A. ${ }^{3} \mathrm{H}$-thymidine uptake was measured at day 3 in breast cancer cells (A, T47D and MCF-7 cells). Similarly, B and C represent ${ }^{3} \mathrm{H}$-thymidine uptake in colorectal (SW620 and DLD-1) and lung (H1299 and H322) cancer lines, respectively.

appears to be independent of tumor histology because breast, colorectal, and lung lines all exhibit substantial growth inhibition.

The effect of Ad-mda7 on five different normal human cell lines (normal human fibroblasts, MJ90; human umbilical vein endothelial cells, HUVEC; normal human melanocytes, NHEM; normal human lung fibroblasts, NHLF; and human mammary epithelial cells, HMEC) were examined. All of them showed no growth inhibition using tritiated thymidine assay when infected with Ad-mda7. However, the tritiated thymidine assay is dependent on the proliferation rate of cells, and many normal cell lines divide very slowly. To further evaluate the effect of Ad-mda7 in normal cells, we used a Trypan blue viability assay and compared with the cytotoxic effects of Ad-mda7 in a panel of normal and tumor cells. As seen in Figure 2A, the primary mammary epithelial HMEC, primary endothelial HUVEC and primary fibroblast cell line MJ90 cells showed minimal cell death when treated with Ad-mda7 or Ad-luc control vector. The other normal cells showed similar results (data not shown). In contrast, tumor cells exhibited substantial cell death after Ad-mda7 treatment (Figure $2 \mathrm{~B}$ ).

\section{Ad-mda7 Induces Apoptosis in Cancer Cells}

Figure $3 \mathrm{~A}$ shows results of a TUNEL assay of MCF-7 cells transduced with Ad-mda7 and Ad-CMVp(A).
These results confirmed that MCF-7 cells were killed via an apoptotic pathway (7). Ad-CMVp(A) or Ad-luc control vectors were ineffective at inducing apoptosis. The colorectal DLD-1 cell line was evaluated for apoptosis induction using Annexin V staining in conjunction with FACS analysis (Figure 3B). Neither the uninfected or Ad-luc-infected (5000 $\mathrm{vp} / \mathrm{cell})$ cells showed substantial levels of apoptosis, whereas Ad-mda7-infected cells exhibited approximately $26 \%$ apoptotic cells at $1000 \mathrm{vp} / \mathrm{cell}$ and $58 \%$ apoptotic cells at $5000 \mathrm{vp} / \mathrm{cell}$. In addition, Ad-mda7 appears to induce apoptosis early after vector infection. Figure 4, $\mathrm{A}$ and $\mathrm{B}$ demonstrate that both NSCLC H1299 and colorectal DLD-1 cells rapidly undergo apoptosis as early as $12 \mathrm{hr}$ postvector treatment (using $1000 \mathrm{vp} /$ cell of Admda7).

The effect of Ad-mda7 on cell-cycle progression was analyzed. Cell-cycle analysis (PI staining) of Ad-mda7 transduced T47D breast cancer cells showed cells undergoing G2/M block and apoptosis (SubG0/Gl phase) in a dose-dependent manner (Figure 4C). Using western blot analyses in conjunction with an anti-BAX antibody we determined that Ad-mda7 infection up-regulated the BAX protein in breast T47D, colorectal DLD-1, and NSCLC $\mathrm{H} 460$ cells in addition to previously reported breast cancer lines (data not shown). Therefore, Ad-mda7 can up-regulate pro-apoptotic BAX protein levels in tu- 
A: Normal Lines

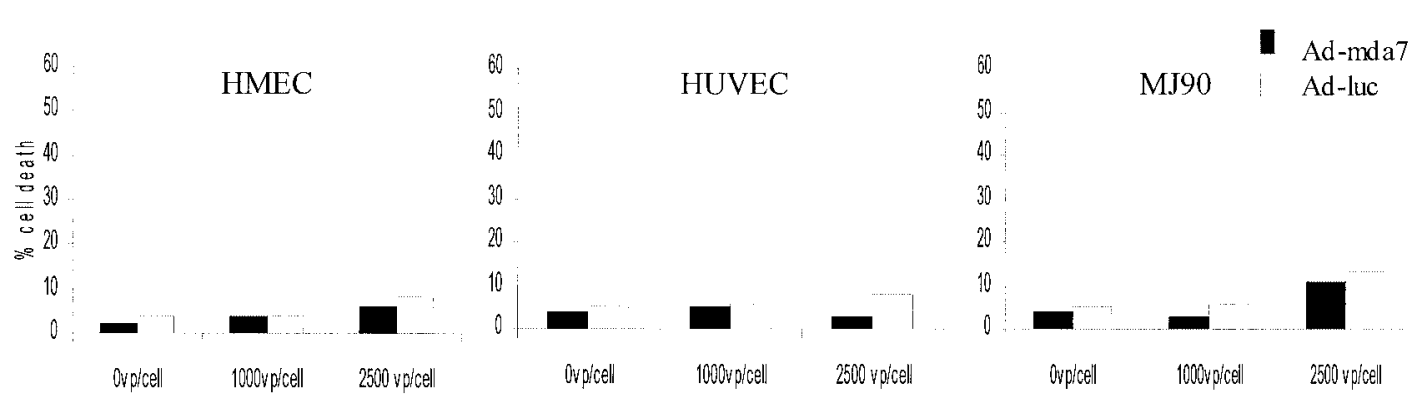

\section{B: Cancer Lines}
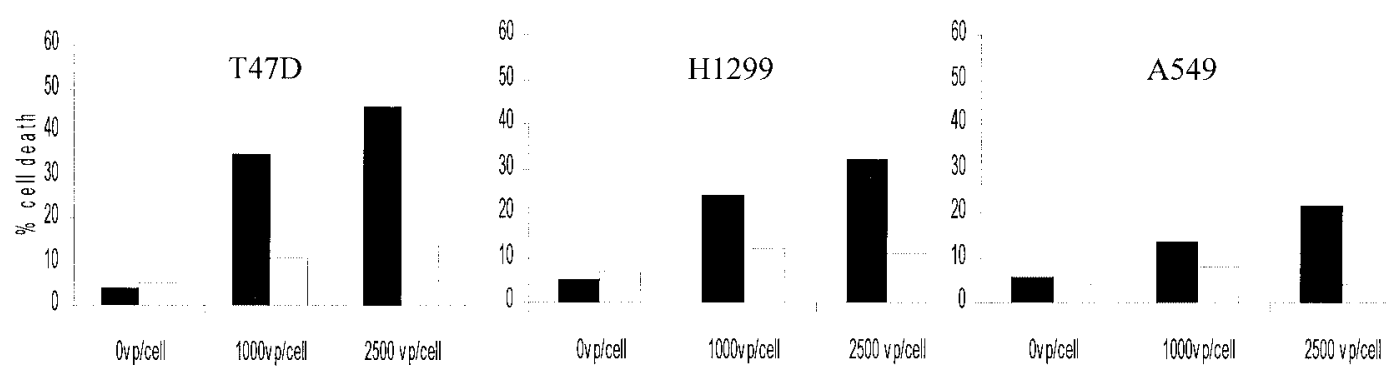

Fig. 2. Effect of Ad-mda7 on normal and cancer cells (Trypan blue assay). Trypan blue exclusion assay was used to evaluate cell death three days post-transduction in normal cells and cancer cells. (A) Human mammary epithelial cells (HMEC), human umbelical vein endothelial cells (HUVEC), and human fibroblasts (MJ90). (B) T47D human breast cancer line, H1299, and A549 non-small-cell lung cancer lines.

mor cells of varying histology. In contrast, we do not see BAX up-regulation in NSCLC H1299 even though these cells are readily killed via apoptosis (measured using Annexin V and TUNEL assays). Similarly, Ad-mda7 induces apoptosis in DU-145 human prostate carcinoma cells, which do not produce BAX protein (8-10).

\section{Ad-mda7-transduced Cancer Cells Secrete Soluble mda-7 Protein}

The subcellular localization of MDA-7 was evaluated in Ad-mda7-transduced NSCLC H460 cells by confocal microscopy. MDA-7 was detected by antiMDA-7 polyclonal antibody conjugated with rhodamine (red staining). Apoptotic cells were also visualized by Annexin V-FITC (green staining) (Figure 5A). The majority of cells expressing MDA-7 were undergoing apoptosis. However, a fraction of cells were undergoing apoptosis that did not appear to be expressing high levels of $\mathrm{MDA}-7$ protein (green stained cells only, shown by arrow). These cells were either undergoing apoptosis due to low levels of MDA-7 expression or were exhibiting apoptosis due to a bystander effector produced by MDA-7 expressing cells. Further confocal analyses of Ad-mda7-transduced H460 cancer cells exhibited a distinctive plasma membrane staining, as well as cytoplasmic, punctate staining, perhaps indicative of secretion of MDA-7 (Figure 5B). This observation was also seen in other cell lines including HeLa (Figure 5C), H1299, T47D, MCF-7 and DLD-1 (data not shown). Supernatants from Ad-mda7 treated cells were analyzed to address whether MDA-7 is actively secreted from the cells. Figure 5D shows the Western blot analysis of cell lysate and supernatant samples obtained from Ad-mda7-infected H1299 cells. The Ad-mda7-transduced cell lysate (lane 1) showed the doublet bands at the predicted sizes of approximately 23 and $17 \mathrm{kD}$. The supernatant sample showed a novel protein band at approximately $40 \mathrm{kD}$ (lane 3). Lysates or supernatants from Ad-lucinfected (lanes 2 and 4) or mock-infected cells did not show any MDA-7 staining.

\section{Genomic Analysis of mda-7 Coding Region}

By genomic database analyses, we found that the mda-7 gene is located on chromosome 1 at 1 q32. The mda-7 gene is encoded by 7 exons, with the $5^{\prime}$ untranslated region being split by an intron (Figure 6). The cDNA for the mda-7 gene was originally isolated and published by Jiang et al. in 1995 (5). This sequence was derived from a human melanotic melanoma cell line, HO-1. More recently, this human mda-7 cDNA sequence has been verified by Soo et al. (26), who isolated cDNA using RT-PCR from an HPV-transformed keratinocyte cell line, HOK. Because both of these DNA sources were tumor cell-derived, we evaluated the mda-7 cDNA se- 
(A)

\section{Ad-mda7}

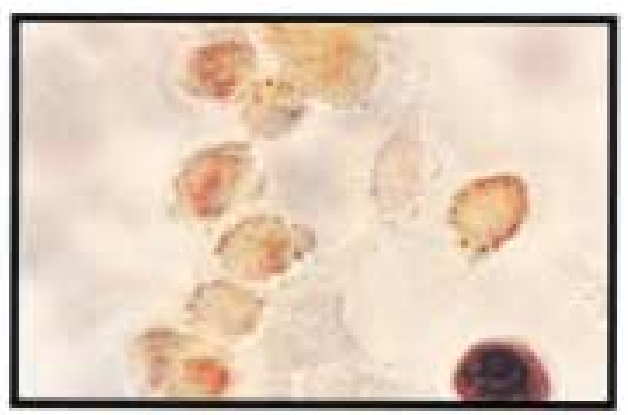

(B)

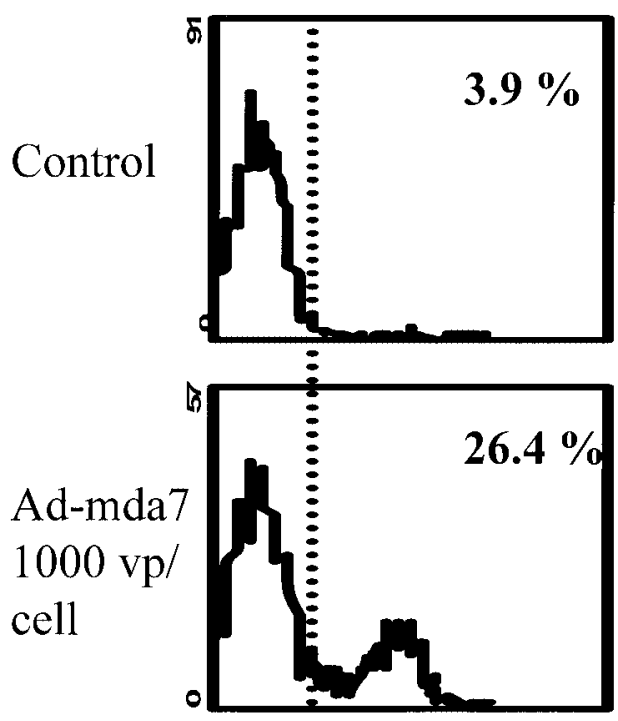

Ad-CMVp(A)
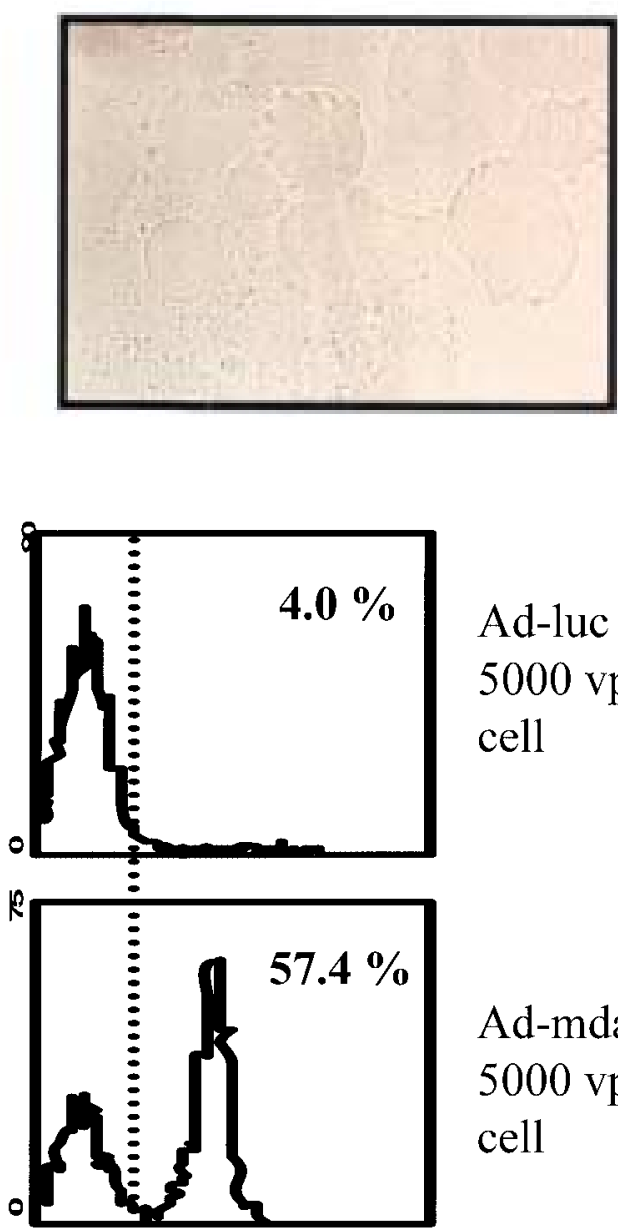

Ad-luc $5000 \mathrm{vp} /$ cell

Ad-mda 7

$5000 \mathrm{vp} /$

cell

Fig. 3. Ad-mda7-induced apoptosis and cell cycle regulation. (A) TUNEL assay: Microscopic illustration of Ad-mda7 infected MCF-7 cells undergoing apoptosis. Ad-CMVp(A) control is shown in the right panel. Apoptotic cells exhibit dark brown staining. (B) DLD-1 cells were infected with Ad-mda7 or Ad-luc and $48 \mathrm{hr}$ later examined for Annexin V staining by FACS analysis. The percentage of positively stained cells is shown.

quence by exon-specific PCR of genomic DNA isolated from a series of PBL from whole blood obtained from 10 normal donors. The mda-7 exons were also sequenced from genomic DNA from a panel of tumor cell lines. The sequences derived from normal blood indicate that the published mda7 cDNA sequences are correct in exons $1,2,3,5$, and 6. However, our analyses of exon 4 indicated a change from the published sequence. In genomic DNA from three PBLs, a homozygous C/C was found corresponding to amino-acid 125. In two samples, homozygous $\mathrm{T} / \mathrm{T}$ was found, whereas the remaining five samples were heterozygous C/T. Genomic DNA from tumor lines from four different tumor types were analyzed and no additional alterations were found (Table 2).

\section{Discussion}

Numerous growth-suppressing and growth-arresting genes (p53, p21/waf, RB, BRCAl, p16, etc.) have been identified and characterized $(4,14-25)$. In this study, we have performed kinetic and dose-dependence analyses on 20 tumor lines from 3 different tumor types and found that 19 of these were strongly growthinhibited by Ad-mda7. Furthermore, Ad-mda7 induced apoptosis in a temporal and dose-dependent fashion in all tumor lines evaluated. In contrast, the five normal cell lines evaluated did not exhibit growth inhibition or cell death after Ad-mda7 treatment.

The overall molecular mechanisms by which Admda7 inhibits growth and induces apoptosis in breast, colorectal and lung cancer cell lines, but not in 
(A)

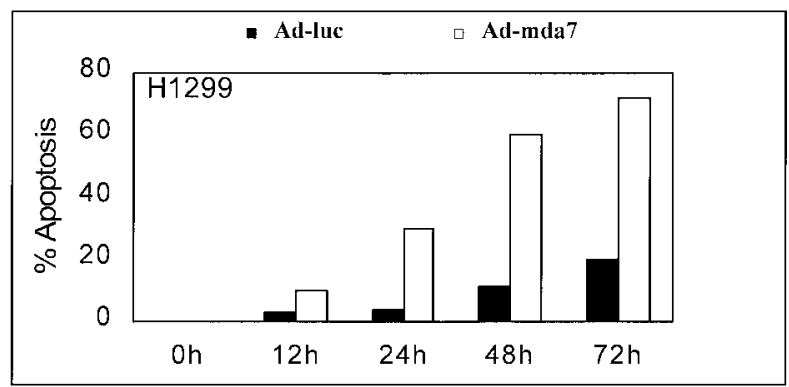

(B)

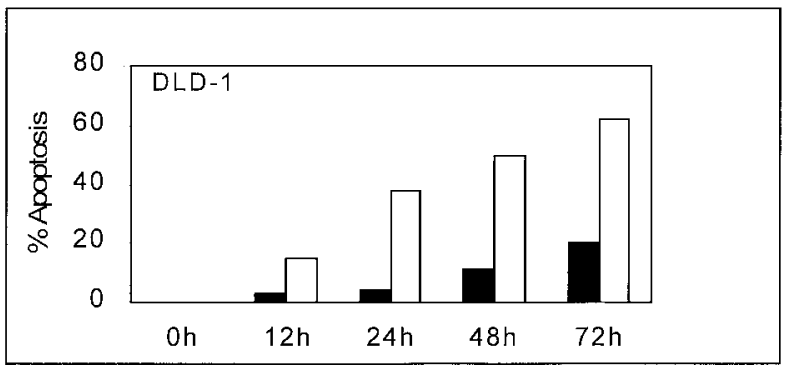

(C)
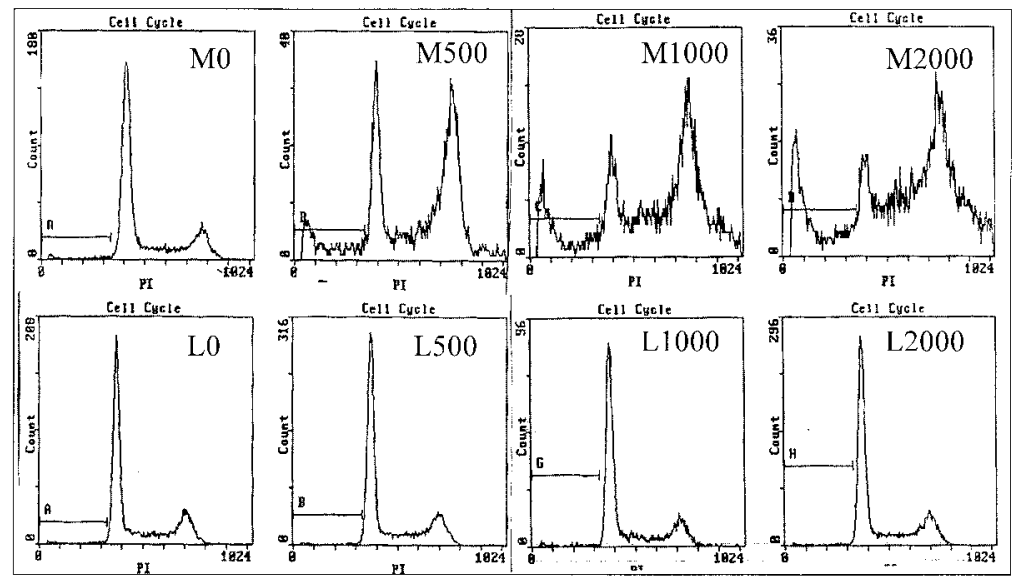

Fig. 4. Ad-mda7-induced rapid apoptosis and cell cycle regulation. Panels A and B shows rapid induction of apoptosis in H1299 and DLD-1 cells infected with Ad-mda7 or Ad-luc, respectively. Cells were evaluated at different time points post-infection for Annexin V-FITC staining by FACS analysis. Panel C shows the cell cycle analysis of Ad-mda7 or Ad-luc transduced T47D breast cancer cells. A dose-dependent increase in G2/M-block and apoptosis (Sub G0/G1) is observed. Increasing MOI (vp/cell) of Ad-mda7 and Ad-luc (control) are denoted as M0, M500, M1000, M2000 and L0, L500, L1000, L2000, respectively.

normal cells, are yet to be defined. However, the growth-inhibitory activity of Ad-mda7 in cancer cells of variable tumor histology and variable p53, RB, p16, and ras genomic status (see Table 1) suggests that MDA-7 triggers novel cellular pathways that lead to cancer growth suppression and apoptosis.

BAX expression is positively regulated by wildtype p53 $(25,28,29)$. The ability of Ad-mda7 to induce BAX appears to be independent of p53, as BAX up-regulation is observed even in p53 mutant cells such as DLD-1, T47D (data not shown). This suggests that the up-regulation of BAX in MDA-7-mediated apoptosis is achieved by both p53-dependent and p53-independent pathways. It should be noted that Ad-mda7 also induces apoptosis in a BAXindependent manner in some cancer cells, including NSCLC H1299 and DU145 (34). Alternatively, the MDA-7 and p53 apoptotic pathways may converge on common downstream apoptotic effectors. Admda7 potently increases steady state levels of p53 protein in $\mathrm{p} 53^{\mathrm{wt}}$ NSCLC A549 and H460 cell lines (34). This may indicate that MDA-7 functions upstream of p53; however, it is not clear whether the increases in p53 protein levels are caused by transcriptional activation of $\mathrm{p} 53$ or posttranscriptional p53 protein stabilization. Because Ad-mda7 induces apoptosis in cells that are mutant for p53 or devoid of p53 or BAX proteins, the up-regulation of p53 and BAX does not appear to be essential for apoptotic induction.

It is interesting to note that Ad-mda7 was able to effectively induce apoptosis in the MCF- 7 breast cancer cells which are deficient in caspase 3, one of the several caspases involved in the downstream apoptotic events. Therefore, Ad-mda7 killing may reflect a novel pathway of apoptosis, which is MDA-7-dependent but independent of caspase 3 and p53. In contrast, the five normal cell lines evaluated were not growth-inhibited after Ad-mda7 transduction. In a separate study, we have found that Ad-mda7 causes activation of caspases 3 and 9 and functions via a BAX-independent mechanism to induce apoptosis in lung cancer cells (34). In addition, Ad-mda7 up-regulates p53 and BAX in p53 wild-type lung cancer cells, again suggesting that MDA-7 signaling may be upstream of p53 (34). We have shown that Ad-mda7 inhibits tumor growth in vivo in NSCLC models (data not shown). In vivo, tumor growth inhibition appears to be due to both induction of apoptosis and anti-angiogenic effects (data not shown).

mda-7 mRNA is down-regulated during melanoma development (5), and MDA-7 protein ex- 

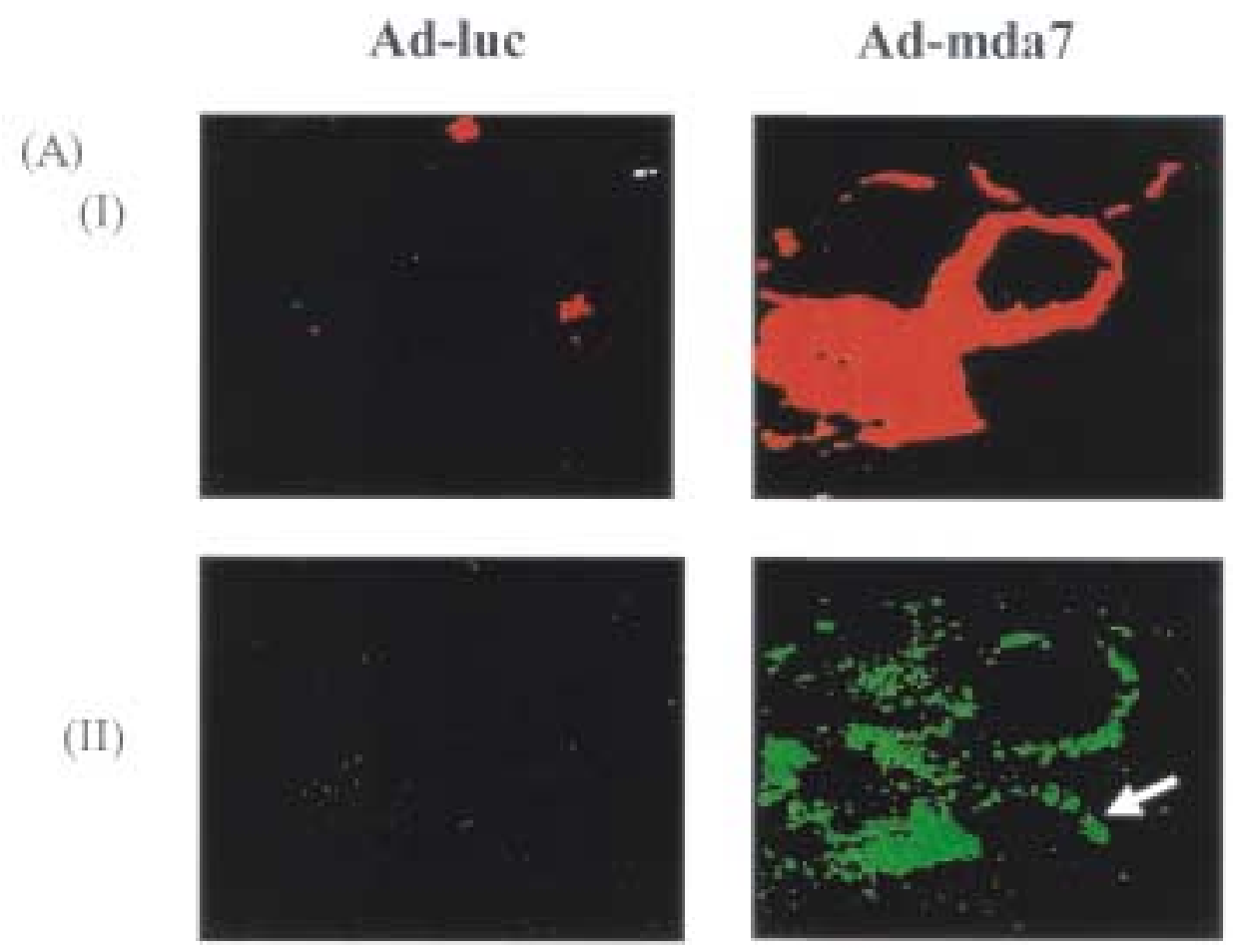

(B)
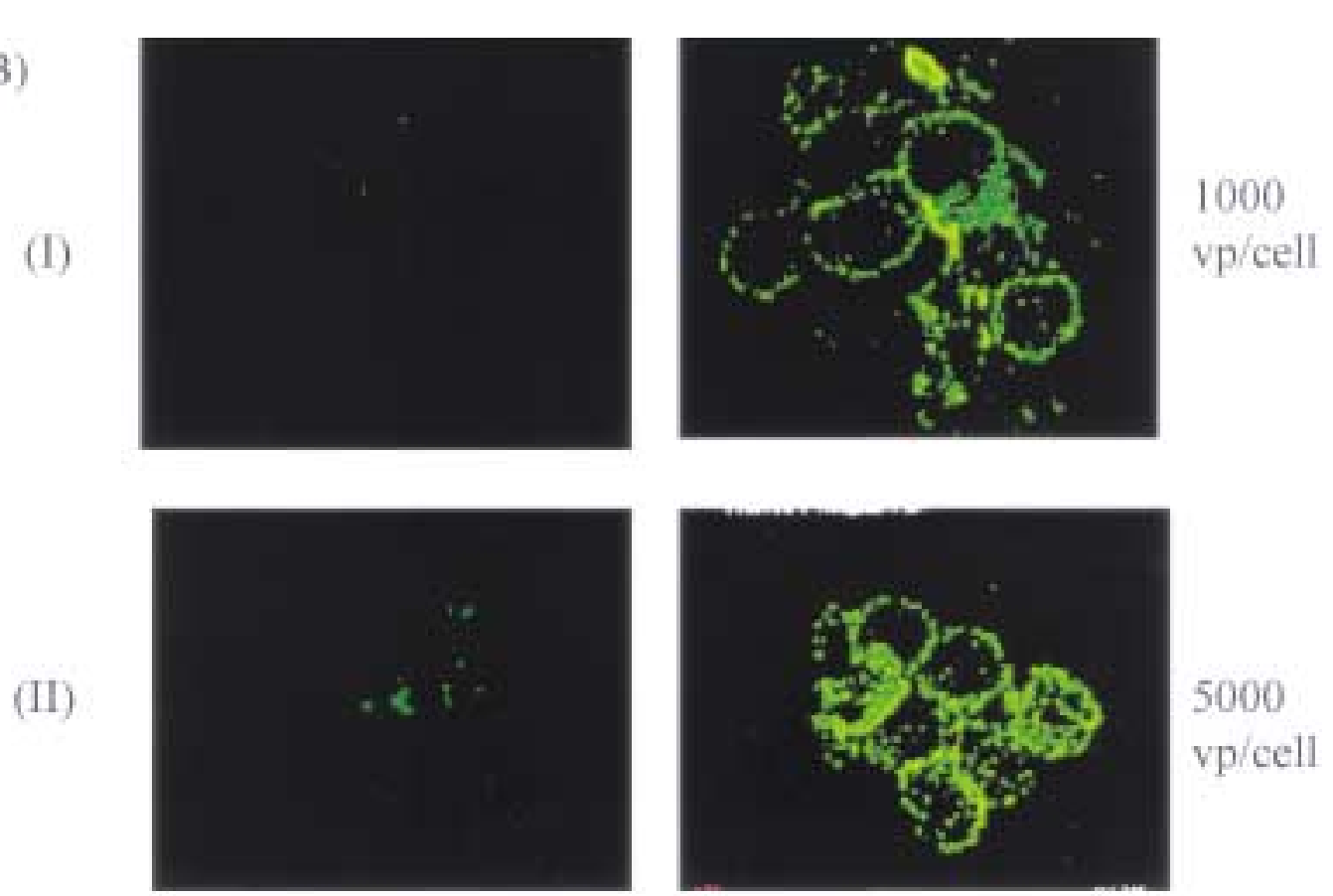

Fig. 5. Confocal analyses of MDA-7 subcellular localization and secretion of soluble MDA-7. (A) Confocal studies. (I) shows surface labeling of MDA-7 protein in H460 cells (red), 48 hours post-Ad-mda7 or Ad-luc infection (MOI of $1000 \mathrm{vp} / \mathrm{cell}$ ). (II) shows the same H460 cells stained with Annexin V-FITC (green ). (B) H460 cells were transduced with 1000 (I) or 5000 (II) MOI of Ad-luc or Ad-mda7, and $48 \mathrm{hr}$ later, cells were incubated with anti-MDA7 rabbit polyclonal antibody followed by goat anti-rabbit IgGtagged FITC (Fluoroscein Iso-thiocynate tag). (C) HeLa cells were infected with $500 \mathrm{vp} / \mathrm{cell}$ of Ad-mda7. The cells were fixed with $2 \%$ para-formaldehyde and treated with $0.001 \%$ Triton X-100 for permeabilization. (I) represents the expression of MDA-7 protein (green fluorescence; using anti-MDA-7 rabbit polyclonal as the primary and anti-rabbit IgG-FITC as the secondary antibody) as a distinct punctate, cytoplasmic staining. (II) represents the same field showing actin fibres stained with Phalloidin Alexa 594. (III) represents the composite of (I) and (II). (D) Western blot analysis of Ad-mda7 transduced H1299 cell lysate (lane 1) and cell supernatant (lane 3); Ad-luc transduced cell lysate (lane 2) and cell supernatant (lane 4) were used as controls. The cells were infected with $1000 \mathrm{vp} / \mathrm{cell}$ of Ad-mda7 or Ad-luc, and $48 \mathrm{hr}$ later, the cell lysate and $0.22 \mathrm{um}$ filtered supernatant were analyzed using the rabbit anti-MDA-7 polyclonal affinity purified antibody. (Figure continued on next page) 

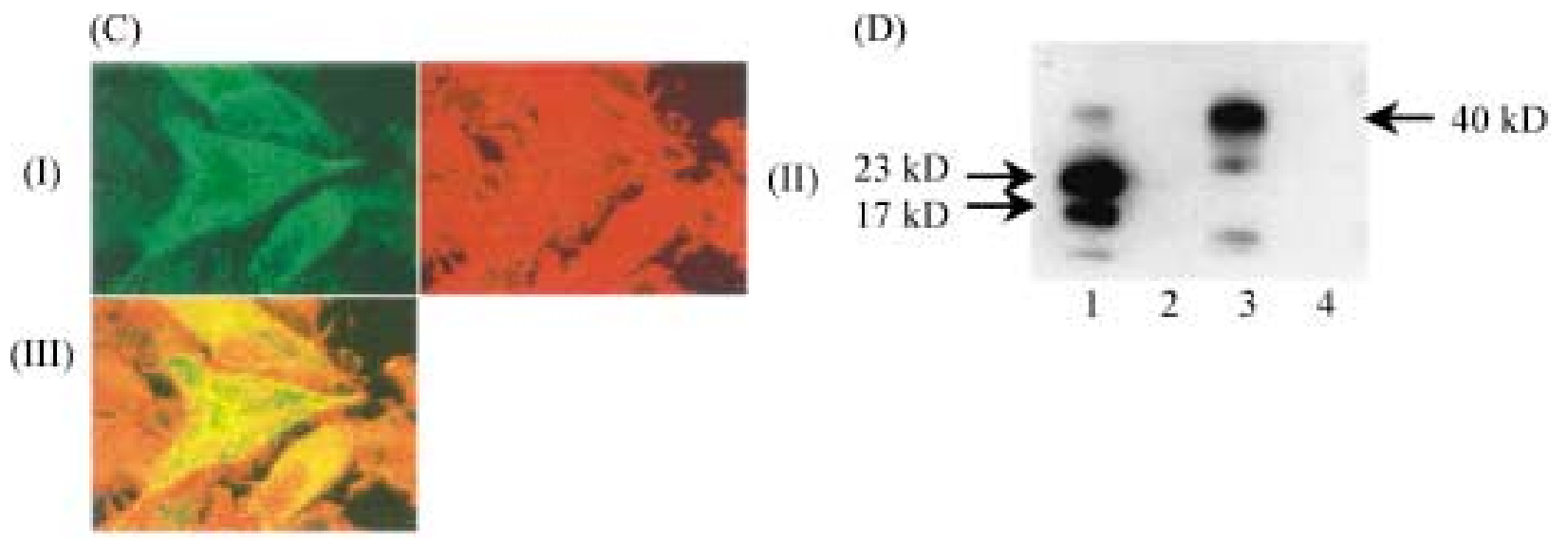

Fig. 5. (Continued).

pression is absent in all the tumor cell lines used in this study. The sub-cellular localization and compartmentalization of transgenic MDA-7 may reveal important clues to its mechanism of action and its interaction with other apoptotic co-factors. Lysates from Ad-mda7-infected tumor and normal cells show an approximate $23 \mathrm{kD} / 17 \mathrm{kDa}$ doublet, indicating that MDA-7 is processed intracellularly. The processing appears to be identical in both normal and tumor cells. Analysis of the MDA-7 primary amino-acid sequence identifies a consensus proteolytic cleavage site after a potential signal sequence. If this signal sequence is used, the cleavage would predict a 23/17 kD doublet. This is consistent with the observed protein bands in the lysate. We do not observe the predicted $17 \mathrm{kD}$ molecule in the supernatant but instead see a dominant $40 \mathrm{kD}$ molecule. Treatment with SDS and boiling suggests that this molecule is not a homo-multimeric form of the pre- dicted $17 \mathrm{kD}$ secreted molecule and further analyses have demonstrated that this $40 \mathrm{kD}$ molecule is heavily glycosylated (data not shown). In a previous study (7), it has been suggested that in human breast cancer cells infected with Ad-mda7, the $23 \mathrm{kD}$ MDA-7 protein translocates from the cytosol to the nucleus. MDA-7 does not possess any consensus nuclear localization motifs, and these authors suggested that MDA-7 protein associates with a cytoplasmic chaperone (such as HMC) $(5,7)$ which might facilitate the translocation of MDA-7 into the nucleus. Our confocal (Figure 5, A-C) and immunohistochemistry studies (data not shown) did not identify nuclear MDA-7 after Ad-mda7 treatment. The ability to selectively and rapidly kill tumor cells and the observation that a secreted form of MDA-7 protein is produced, which may possess bystander tumorsuppressive activity, makes Ad-mda7 a promising candidate for anti-cancer gene therapy.

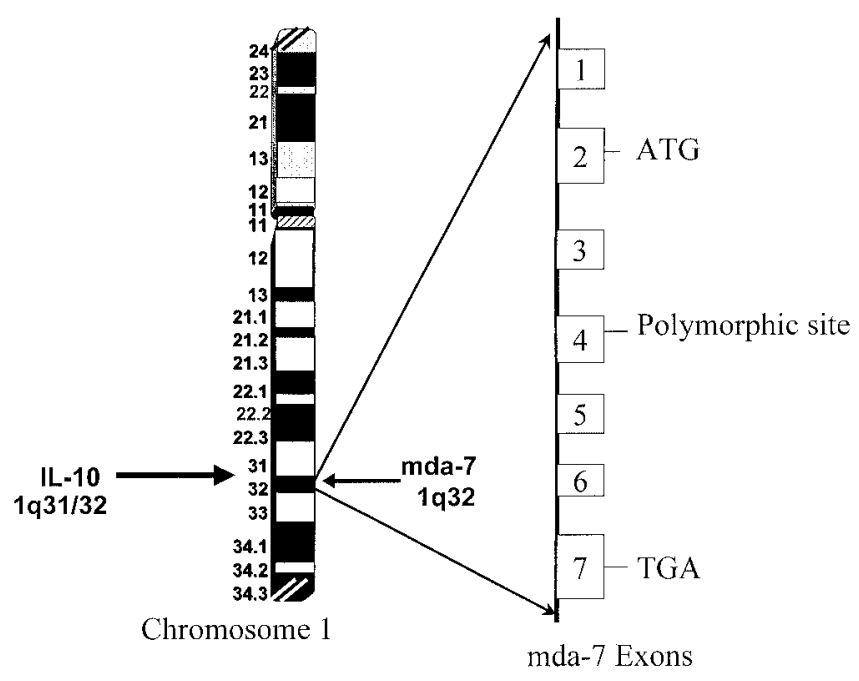

Fig. 6. Schematic representation of chromosome location of mda7 and exon map of the mda7 locus. 
Table 1. Status of tumor suppressor gene/oncogene/cell-cycle regulation genes (p53, RB, p16, ras) in normal and cancer cell types used in this study and their correlation with Ad-mda7 killing

\begin{tabular}{|c|c|c|c|c|c|}
\hline Cell Type & Ad-mda7 Killing & p53 & $\mathbf{R B}$ & Ras & p16 \\
\hline \multicolumn{6}{|l|}{ NORMAL } \\
\hline Hmec & - & wt & wt & wt & wt \\
\hline MJ90 fibroblasts & - & wt & wt & wt & wt \\
\hline HuVEC & - & wt & wt & wt & wt \\
\hline \multicolumn{6}{|l|}{ BREAST } \\
\hline T47D & + & Mut & - & wt & - \\
\hline MCF-7 & + & wt & - & wt & - \\
\hline \multicolumn{6}{|l|}{ LUNG } \\
\hline H1299 & + & Null & wt & Mut & Neg \\
\hline A549 & + & wt & - & Mut & Neg \\
\hline $\mathrm{H} 460$ & + & wt & wt & Mut & Del \\
\hline \multicolumn{6}{|l|}{ COLORECTAL } \\
\hline SW620 & + & Mut & - & Mut & Neg \\
\hline НCT116 & + & wt & - & Mut & Neg \\
\hline
\end{tabular}

To date, many different tumor suppressor genes encoding functionally diverse proteins have been identified $(3,14,15,16,17)$. The earliest tumor suppressors identified encoded nuclear trans-activators such as p53 and Rb. Subsequently, cytoplasmic and membrane-bound tumor suppressor genes have been identified. More recently, secreted tumor suppressors have also been identified and characterized and mda-7 appears to be a novel member of this category. Gene therapeutics developed using secreted tumor suppressors should have greater anti-tumor effects than those encoding nuclear or cytoplasmic tumor suppressors. It is anticipated that secreted tumor suppressors will have a greater "radius of effect" owing to their ability to affect neighboring cells. The best-characterized secreted tumor suppressor is maspin, a member of the large serpin family of serine protease inhibitors (30). Maspin expression is down-regulated in breast and prostate cancer. Overexpression of maspin suppresses the growth and metastasis of breast cancer cells in vivo and appears to play a role in angiogenesis (30). Overexpression of uteroglobin (UG), another secreted tumor suppressor, also reverses the anchorage independence and extracellular matrix invasion characteristics of the transformed phenotype (31). In addition, in vitro transformation of primary cultures of chick embryo fibroblasts by $\mathrm{v}$-Src or $\mathrm{v}$-Jun has been correlated

Table 2. Genomic analyses of mda-7 exons in tumor cells and normal blood PBL

\begin{tabular}{|c|c|c|c|c|}
\hline Cell Line & Origin & p53 Status & $\begin{array}{c}\text { Exon } 4 \\
\text { Polymorphism }\end{array}$ & $\begin{array}{c}\text { Ad-mda7 } \\
\text { Growth Inh. }\end{array}$ \\
\hline A549 & NSCLC & wt & $\mathrm{C} / \mathrm{T}$ & + \\
\hline H1299 & NSCLC & null & $\mathrm{C} / \mathrm{T}$ & + \\
\hline DLD-1 & Colorectal & mut & $\mathrm{C} / \mathrm{C}$ & + \\
\hline PC-3 & Prostate & null & $\mathrm{C} / \mathrm{C}$ & + \\
\hline HeLa & Cervical & wt & $\mathrm{C} / \mathrm{T}$ & + \\
\hline $\begin{array}{l}\text { PBL } \\
(n=5)\end{array}$ & $\begin{array}{l}\text { Normal } \\
\text { blood }\end{array}$ & wt & $\mathrm{C} / \mathrm{C}$ & n.d. ${ }^{1}$ \\
\hline $\begin{array}{l}\text { PBL } \\
(n=3)\end{array}$ & $\begin{array}{l}\text { Normal } \\
\text { blood }\end{array}$ & wt & $\mathrm{C} / \mathrm{T}$ & n.d. \\
\hline $\begin{array}{l}\text { PBL } \\
(n=2)\end{array}$ & $\begin{array}{l}\text { Normal } \\
\text { blood }\end{array}$ & wt & $\mathrm{T} / \mathrm{T}$ & n.d. \\
\hline
\end{tabular}

${ }^{1}$ n.d. : not done 
with the down-regulation of the secreted protein, Acidic and Rich in Cysteine (SPARC) (32). Overexpression of SPARC in the transformed cultures reduces their capacity to develop local fibrosarcomas in vivo. Recently, Melkonyan et al. (33) identified a human secreted apoptosis-related protein 2 (SARP2) with pro-apoptotic effects, that is down-regulated in breast cancer lines. Preliminary data suggest that secreted MDA-7 may have growth-inhibitory activity on tumor cell lines in vitro (data not shown). In other studies, we have shown that Ad-mda7 inhibits endothelial cell differentiation in vitro and exhibits anti-angiogenic properties in vivo (data not shown).

A comparison of the mda-7 cDNA with other database sequences reveals, at the DNA level, strong similarity to rat c49a $(63 \%$ identity overall in the coding region; $82 \%$ identity over a stretch of $476 \mathrm{nu}-$ cleotides). It is believed that $\mathrm{c49}$ a is the rat homolog of human MDA-7. c49a was cloned by virtue of its being up-regulated during wound healing; however, no function has been attributed to this novel gene (26). An independent isolate of c49a was termed mob-5, which encoded a $23 \mathrm{kD}$ cytokine-like secreted protein, whose expression could be induced by oncogenic $\mathrm{H}$-ras and $\mathrm{K}$-ras but not by normal ras activation (27).

The exon 4 polymorphism at residue 125 results in a change in codon usage, and this position can encode either a His or a Tyr residue (Table 2). The published and Genbank mda-7 cDNA sequences encode a His at aa 125. These results indicate that this is a polymorphic site rather than a disease-associated mutation. The role of the exon 4 polymorphism, found in both normal and tumor cells, is yet to be defined. The recent paper by Zhang et al. (27) also sequenced rat and human mda-7 cDNAs from tumor sources. The human mda-7 cDNA was reported to contain a single amino acid insertion (serine 15) in the signal peptide of the published mda-7 sequence. We did not observe such genetic alteration after sequencing all mda-7 exons from more than 15 different genomic sources. The lack of mutations in the mda-7 coding region in tumor cell lines suggests that this gene may not be mutated similarly to "classical" tumor suppressor genes. Note that since only exon-specific sequencing was performed, we cannot exclude the possibility of mutations in other regions of the gene locus.

By genomic database analyses, we have found that the mda-7 gene is located on chromosome 1 at $1 \mathrm{q} 32$ (Figure 4). Chromosome $\mathrm{lq}$ is known to be altered in lung, breast, and renal tumors (35-38). The mda-7 gene locus is located close to the gene encoding the cytokine IL-10 (at 1q31/32). Protein sequence analyses suggest that MDA-7 is a distant member of the IL-10 family of secreted proteins. Although, the MDA-7 protein displays only limited homology to human IL- 10 (54\% in a 42 aminoacid stretch and $19 \%$ overall identity), MDA-7 does appear to share the 6-helix structural configuration of IL-10 family members (data not shown). Addi- tional family members have recently been cloned, including IL-19 (39), IL-TIF (IL-22), (40), AK 155 (41), and IL-20/ ZCYTO10 (Accession number: AAF36679. Conklin D, Grossmann A, Haldeman B, O'Hara P. Four alpha helix cytokine). IL-10 protein functions as a secreted non-glycosylated dimer, whereas MDA-7 is heavily glycosylated. Ad-mda7 is anti-angiogenic in vitro and in vivo, another feature in common with IL-10; however, Ad-mda7 does not appear to share the immunosuppressive properties of IL-10 (data not shown). Therefore, mda7 may represent a secreted apoptosis-inducing gene with limited homology to cytokines. Further studies are ongoing to characterize, at a molecular level, the anti-tumor effects of Ad-mda7.

\section{Acknowledgements}

This research was supported by Introgen Therapeutics Inc., Houston, TX. and NCI grant R43 CA86587 to SC.

\section{References}

1. Kozarsky K, Wilson J. (1993) Gene therapy. Adenovirus vectors. Curr. Opin. Gene Dev. 3: 499-503.

2. Swisher S, Roth J, Nemunaitis, Lawrence D, Kemp B, Carrasco C, Connors D, El-Naggar A, Fossella F, Glisson B, Hong W, Khuri F, Kurie J, Lee J, Lee J, Mack M, Merritt J, Nguyen D, Nesbitt J, Perez-Soler R, Pisters K, Putnam J Jr, Richli W, Savin M, Waugh M. (1999) Adenovirus-mediated p53 gene transfer in advanced non-small-cell lung cancer. J. Natl. Cancer Inst. 91: 763-771.

3. White E. (1996). Life, death and the pursuit of apoptosis. Genes Development 10: 1-15.

4. Jiang H, Fisher P. (1993) Use of a sensitive and efficient subtraction hybridization protocol for the identification of genes differentially expressed during growth, differentiation in human melanoma cells. Mol. Cell Different. 1: 285-299.

5. Jiang H, Lin J, Su Z, Goldstein N, Fisher P. (1995) Subtraction hybridization identifies a novel melanoma differentiation associated gene, mda-7, modulated during human melanoma differentiation, growth and progression. Oncogene. 11: 2477-2486.

6. Jiang H, Su Z, Lin J, Goldstein N, Fisher, P. (1996) The melanoma differentiation associated gene mda-7 suppresses cancer cell growth. Proc. Natl. Acad. Sci. 93: 9160-9165.

7. Su Z, Madireddi M, Lin J, Young C, Kitada S, Reed J, Goldstein N, Fisher P. (1998) The cancer growth suppressor gene mda-7 selectively induces apoptosis in human breast cancer cells and inhibits tumor growth in nude mice. Proc. Natl. Acad. Sci. 95: 14400-14405.

8. Su Z, Madireddi M, Young C, Jiang H, Goldstein N, Fisher P. (2000) Melanoma differentiation associated gene-7 (Mda-7): a ubiquitous cancer growth suppressor gene. Cancer Gene Therapy (in press).

9. Madireddi M, Su Z, Young C, Goldstein N, Fisher P. (2000) Mda-7, a novel melanoma differentiation associated gene with promise for cancer gene therapy. Cancer Gene Therapy: Past Achievements and Future Challenges (in press).

10. Madireddi M, Su Z, Young C, Goldstein N, Fisher P. (2000) Mda-7, a novel melanoma differentiation associated gene with promise for cancer gene therapy. Adv. Exp. Med. Biol. 465: 239-61.

11. Nielsen L, Maneval D. (1998) p53 tumor suppressor gene therapy for cancer. Cancer Gene Therapy. 5: 52-63.

12. Casey G, Lo-Hsueh M, Lopez M, Vogelstein B, Stanbridge E. (1991) Growth suppression of human breast cancer cells by the introduction of a wild-type p53 gene. Oncogene. 6: 1761-1797. 
13. Graham F, Prevec. (1992) Adenovirus-based expression vectors and recombinant vaccines. Biotechnology. 20: 363-390.

14. Jiang H, Lin J, Su Z, Collart F, Huberman E, Fisher P. (1994) Induction of differentiation in human promyelocytic HL-60 leukemia cells activates p21, WAF1/CIP1, expression in the absence of p53. Oncogene. 9: 3397-3406.

15. Jiang H, Lin J, Su Z, Kerbel R, Herlyn M, Weissman D, Welch D, Fisher P. (1995) The melanoma differentiation associated gene MDA-6, which encodes the cyclin-dependent kinase inhibitor p2 1 , is differentially expressed during growth, differentiation and progression in human melanoma cells. Oncogene. 10: 1855-1864.

16. Schneider C, King R, Philipson L. (1988) Genes specifically expressed at growth arrest of mammalian cells. Cell 54: 787-793.

17. Fornace A Jr, Alamo I Jr. HollanderM. (1988) DNA damageinducible transcripts in mammalian cells. Proc. Natl. Acad. Sci. 85: 8800-8804.

18. Abdollahi A, Lord K, Hoffman-Liebermann B, Liebermann D. (1991) Sequence and expression of cDNA encoding MyD118: a novel myeloid differentiation primary response gene induced by multiple cytokines Oncogene. 6: 165-167.

19. Mooslehner K, Muller U, Karls U, Hamann L, Harbers K. (1991) Structure and expression of a gene encoding a putative GTP-binding protein identified by provirus integration in a transgenic mouse strain. Mol. Cell Biol. 11: 886-893.

20. Del Sal G, Murphy M, Ruaro E, Lazarevic D, Levine A, Schneider C. (1995) Cyclin D1 and p21/wafl are both involved in p53 growth suppression. Oncogene. 12: 177-185.

21. Wu G, El-Diery W. (1996) p53 and chemosensitivity. Nat. Med. 2: 255-256.

22. Claudio P, Howard C, Baldi A, De Luca A, Fu Y, Condorelli G, Sun Y, Colburn N, Calabretta B, Giordano A. (1994) p130/pRb2 has growth suppressive properties similar to yet distinctive from those of retinoblastoma family members $\mathrm{pRb}$ and p107. Cancer Res. 54: 5556-5565.

23. Noda M, Kitayama H, Matsuzaki T, Sugimoto $Y$, Okayama H, Bassin R, Ikawa Y. (1988) Detection of genes with a potential for suppressing the transformed phenotype associated with activated ras genes. Proc Natl. Acad. Sci. 86: 162-166.

24. Kitayama H, Sugimoto Y, Masuzaki T, Ikawa Y, Noda M. (1989) A ras-related gene with transformation suppressor activity. Cell 56: 77-84.

25. Reed J, Zha J, Aime-Sempe C, Takayama S, Wang, H. (1996) Structure-function analysis of Bcl-2 family proteins. Regulators of programmed cell death. Adv. Exp. Med. Biol. 406: 99-112.

26. Soo C, Shaw W, Freymiller E, Longaker M, Bertolami C, Chie R, Tieu A, Ting K. (1999) Cutaneous rat wounds express c49a, a novel gene with homology to the human melanoma differentiation associated gene, MDA-7. J. Cell. Biochem. 74: 1-10.

27. Zhang R, Tan Z, Liang P. (2000) Identification of a novel ligand-receptor pair constitutively activated by ras oncogenes. $J$. Biol. Chem. 11: 24436-24443.
28. Reed J. (1997) Double identity for proteins of the Bcl-2 family. Nature 387: 773-776.

29. Sedlak T, Oltvai Z, Yang E, Wang K, Boise L, Thompson C, Korysmeyer S. (1995) Multiple Bcl-2 family members demonstrate selective dimerizations with Bax. Proc. Natl. Acad. Sci. 92: 7834-7838.

30. Umekita Y, Hiipakka R, Liao S. (1997) Rat and human maspins: structures, metastatic suppressor activity and mutation in prostate cancer cells. Cancer Letter 126: 87-93.

31. Mukherjee A, Kundu G. Mantile-Selvaggi G, Yuan C, Mandal A, Chattopadhyay S, Zheng F, Pattabiraman N, Zhang Z. (1999) Uteroglobin: a novel cytokine? Cell Mol. Life. Sci. 55: 771-87.

32. Vajkoczy P, Menger M, Goldbrunner R, Ge S, Fong T, Vollmar B, Schilling L, Ullrich A, Hirth K, Tonn J, Schmiedek P, Rempel S. (2000) Int. J. Cancer. 87: 261-268.

33. Melkonyan H, Chang W, Shapiro J, Mahadevappa M, Fitzpatrick P, Kiefer M, Tomei L, Umansky S. (1997) SARPs: a family of secreted apoptosis-related proteins. Proc. Natl. Acad. Sci. 94:13636-13641.

34. Saeki T, Mhashilkar A, Roth J, Chada S, Ramesh R. (2000) Tumor-suppressive effects by adenovirus-mediated mda-7 gene transfer in non-small cell lung cancer cell in vitro. Gene Ther. 7: 2051-2057.

35. Bieche I, Champeme M, Lidereau R. (1995) Loss and gain of distinct regions of chromosome $\mathrm{lq}$ in primary breast cancer. Clin. Cancer Res. 1: 123-127.

36. Gronwald J, Storkel S, Holtgreve-Grez H, Hadaczek P, Brinkschmidt C, Jauch A, Lubinski J, Cremer T. (1997) Comparison of DNA gains and losses in primary renal clear cell carcinomas and metastatic sites: importance of $1 q$ and $3 p$ copy number changes in metastatic events. Cancer Res. 57: 481-487.

37. Petersen I, Bujard M, Petersen S, Wolf G, Goeze A, Schwendel A, Langreck H, Gellert K, Riechel M, Just K, Du Manoir S, Cremer T, Dietel M, Ried T. (1997) Patterns of chromosomal imbalances in adenocarcinoma and squamous cell carcinoma of the lung. Cancer Res. 57: (12): 2331-2335.

38. Sukamoto K, Yoshimoto M, Kasumi M, Akiyama F, Sakamoto G, Nakamura Y, Emi M. (1999) Frequent multiplication of chromosome $\mathrm{lq}$ in non-invasive and papillotubular carcinoma of the breast. Cancer Letter. 141: 21-27.

39. Gallagher G, Dickensheets H, Eskdale J, Izotova L, Mirochnitchenko O, Peat J, Vazquez N, Pestka S, Donnelly R, Kotenko S. (2000) Cloning, expression and initial characterization of interleukin 19 (IL-19), a novel hoomologue of human IL-10 (IL-10). Genes and Immunity 1: 442-450.

40. Dumoutier L, Louahed J, Renauld J. (2000) Cloning and characterization of IL-10-related T cell-derived inducible factor (IL-TIF), a novel cytokine structurally related to IL-10 and inducible by IL-9. J. Immunol. 164: 1814-9.

41. Knappe A, Hor S, Wittmann S, Fickenscher H. (2000) Induction of a novel cellular homolog of interleukin-10, AK 155, by transformation of $\mathrm{T}$ lymphocytes with herpesvirus saimiri. J. Virol. 74: 3881-3887. 